CORRIGENDUM

doi:10.1038/nature10789

\title{
Selective killing of cancer cells by a small molecule targeting the stress response to ROS
}

Lakshmi Raj, Takao Ide, Aditi U. Gurkar, Michael Foley, Monica Schenone, Xiaoyu Li, Nicola J. Tolliday, Todd R. Golub, Steven A. Carr, Alykhan F. Shamji, Andrew M. Stern, Anna Mandinova, Stuart L. Schreiber \& Sam W. Lee

Nature 475, 231-234 (2011)

In this Letter we made errors in the description of the way we performed tumour measurements in the spontaneous (Fig. 2b and d) and the xenograft (Supplementary Fig. 9) mouse models. The legend of Fig. 2 incorrectly states that the graph of plotted tumour measurements represents tumour diameters from "grossly dissected tumours". Similarly, the Supplementary Fig. 9c legend incorrectly states that "After 3 weeks, tumour masses were excised and measured" and the legend of Supplementary Fig. 9d incorrectly states that: "After 3 weeks treatment, tumour sizes were measured". Instead, for the experiments in both Fig. 2 and Supplementary Fig. 9 we measured diameters of tumours in live mice at different time points (as indicated on the $x$ axis of the graphs) during the course of the treatment. The tumours were measured between the skin surface layers using a caliper.

Additionally, the legend of Supplementary Fig. 9b and c incorrectly refers to "tumour mass(es)" instead of "tumour size(s)". In the MMTV-PyVT spontaneous tumour model (Fig. 2b and d) and in the xenograft bladder, breast and lung models (Supplementary Fig. 9b, c and d) we measured the length and the width of the tumour. The length was measured along the imaginary longitude of the corpse of the mouse
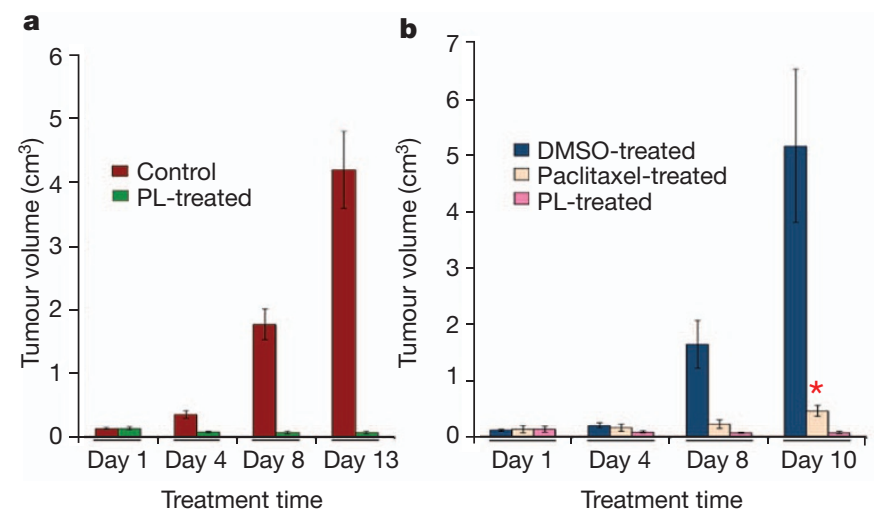

Figure $1 \mid$ Additional data (volume measurements) for Fig. $2 b$ and $d$ of the original paper. Tumour volume measurement in MMTV-PyVT mice. a, Tumour volume of single tumours from control (dimethyl sulphoxide, DMSO) or piperlongumine (PL)-treated mice were calculated on the basis of the length and width of the legions after applying the following formula: length $\times$ width $^{2} \times \pi / 6$. b, Similarly, tumour volumes were calculated from DMSO-, paclitaxel- or PL-treated mice. Values in bar graphs are mean \pm standard deviation $(n=12)$. *Shorter treatment period due to high toxicity of paclitaxel in animals. and the width was measured in the direction of its latitude. In the melanoma xenograft model (Supplementary Fig. 9e) we measured only the width owing to the irregular longitudinal shape of the tumours. For consistency, the graphs in Fig. 2 and Supplementary Fig. 9 represent only the width measurements.

Furthermore, we defined the error bars in Fig. 2 incorrectly: the values in bar graphs in Fig. $2 b$ and $d$ are mean \pm standard deviation $(n=12)$. We also realize that these error bars were misleadingly small when compared to calculated tumour volumes (in cubic centimetres) using the following formula: length $\times$ width $^{2} \times \pi / 6$. Tumour volume measurements of control and treated single tumours in the MMTVPyVT mouse model are shown below (Fig. 1 of this Corrigendum). Although we did observe much higher variability in tumour volumes than in tumour width, the magnitude of the difference between control and treated animals remains the same. In addition, we made errors in indicating the exact number of animals used for each experiment in Supplementary Fig. 9. The correct numbers are 12 animals per group for Supplementary Fig. 9b and seven animals per group for Supplementary Fig. 9c, d and e. Values in Supplementary Fig. 9 are averages ( \pm standard deviation; $n=12$ for Supplementary Fig. 9b, $n=7$ for Supplementary Fig. 9c, d and e).

We have also been unable to verify without doubt that the image in Supplementary Fig. 9b shows four different mice within the treated and untreated groups and therefore wish to replace this figure (see Fig. 2 of this Corrigendum).

We apologize for any confusion these errors may have caused. The corrections described here do not alter the overall conclusions of this Letter, and all other data still stand.

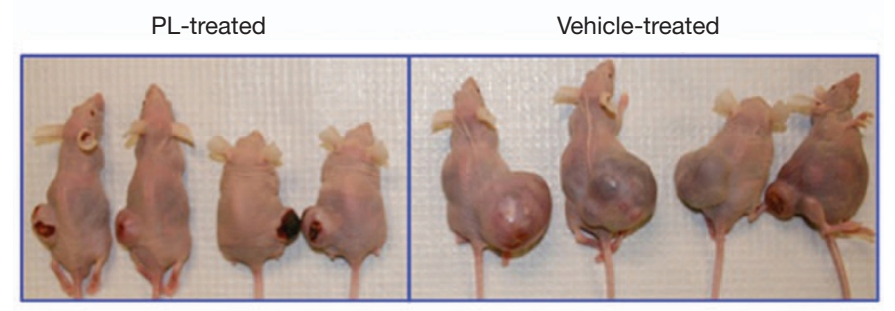

Figure $2 \mid$ This is the corrected Supplementary Fig. 9b. Therapeutic activity of PL in xenograft tumour mice bearing EJ bladder cancer cells. Control (vehicle)- and PL-treated mice were killed after six weeks of treatment. Treatment was $1.5 \mathrm{mg} \mathrm{kg}^{-1}$ of PL by intraperitoneal injection daily. 